Bull. Korean Math. Soc. 49 (2012), No. 6, pp. 1241-1250

http://dx.doi.org/10.4134/BKMS.2012.49.6.1241

\title{
MAXIMUM PRINCIPLE AND COMPARISON PRINCIPLE OF $p$-HARMONIC FUNCTIONS VIA $p$-HARMONIC BOUNDARY OF GRAPHS
}

\author{
Yong HaH LeE
}

\begin{abstract}
We prove the maximum principle and the comparison principle of $p$-harmonic functions via $p$-harmonic boundary of graphs. By applying the comparison principle, we also prove the solvability of the boundary value problem of $p$-harmonic functions via $p$-harmonic boundary of graphs.
\end{abstract}

\section{Introduction}

The maximum principle and the comparison principle are interesting topics in studying the behavior of solutions of some equations. In particular, the maximum principle and the comparison principle enable us to control the behavior of solutions of a certain equation by the boundary data of the solutions. Such a study has been developed for several equations. For instance, Holopainen and Soardi [1] proved the maximum principle and the comparison principle of $p$-harmonic functions on finite subsets of graphs. Later, Kim and Chung [2] generalized the result of Holopainen and Soardi by extending the result into infinite subsets. However, those results are related to the real boundary, not the ideal boundary like infinite boundary. In fact, in the case that a given graph has no boundary, we need to lean on the ideal boundary in controlling the behavior of solutions at infinity of the graph. In line with the viewpoint, we rebuild the maximum principle and the comparison principle to control the whole behavior of $p$-harmonic functions on the graphs.

In this paper, we newly suggest the maximum principle and the comparison principle of $p$-harmonic functions on a graph, in such a way that they are described in terms of the $p$-harmonic boundary as follows:

Received September 29, 2010; Revised April 5, 2012.

2010 Mathematics Subject Classification. 31C20, 60J50, 94C15.

Key words and phrases. maximum principle, comparison principle, $p$-harmonic function, $p$-harmonic boundary, boundary value problem.

This research was supported by Basic Science Research Program through the National Research Foundation of Korea(NRF) funded by the Ministry of Education, Science and Technology (2009-0073057). 
Theorem 1.1. Let $G$ be a graph of bounded degree and $u, v \in \mathcal{H B D}_{p}(G)$. Then the followings hold:

(i) (Maximum principle) If $a \leq u \leq b$ on $\Delta_{p, G}$ for some constants a and $b$, then $a \leq u \leq b$ on $V_{G}$.

(ii) (Comparison principle) If $v \leq u$ on $\Delta_{p, G}$, then $v \leq u$ on $V_{G}$.

This result is a direct generalization of that of Holopainen and Soardi [1]. As an application of the comparison principle, we also prove the solvability of the boundary value problem of $p$-harmonic functions in terms of $p$-harmonic boundary of graphs as follows:

Theorem 1.2. Let $G$ be a graph of bounded degree. Then for any continuous function $f$ on $\Delta_{p, G}$, there exists a unique p-harmonic function $h$, which is a limit of a sequence of functions in $\mathcal{H B D}_{p}(G)$ in the sense of supremum norm, such that

for all $\mathbf{x} \in \Delta_{p, G}$.

$$
h(\mathbf{x})=f(\mathbf{x})
$$

\section{Preliminaries}

Let $G=\left(V_{G}, E_{G}\right)$ be an infinite graph with no self-loops, where $V_{G}$ and $E_{G}$ denote the set of all vertices and the set of all edges of $G$, respectively. If vertices $x, y$ in $V_{G}$ are the endpoints of a same edge, then we say that they are neighbors, and write $y \sim x$ and $x \sim y$. The degree of $x$ is the number of all neighbors of $x$. If the degrees of vertices of a graph $G$ are uniformly bounded, then $G$ is called a graph of bounded degree.

A sequence $\left(x_{0}, x_{1}, \ldots, x_{n}\right)$ of vertices in $V_{G}$ is called a path from $x_{0}$ to $x_{n}$ with the length $n$ if $x_{k} \sim x_{k-1}$ for each $k=1,2, \ldots, n$. A subset $U$ of $V_{G}$ is said to be connected if any two vertices in $U$ are can be joined by a path in $U$. We define the distance $d(x, y)$ between two vertices $x$ and $y$ to be the minimum of the lengths of paths from $x$ to $y$. Then $d$ defines a metric on $V_{G}$. For this metric $d$, define an $n$-neighborhood $N_{n}(x)=\left\{y \in V_{G}: d(x, y) \leq n\right\}$ for each $x \in V_{G}$ and for each $n \in \mathbb{N}$. From now on, all the graphs considered in this paper are connected infinite with no self loops and of bounded degree, unless otherwise specified.

Let $u$ be a real valued function defined on $S \cup \partial S$, where $\partial X=\left\{x \in V_{G}\right.$ : $d(x, X)=1\}$ for each subset $X$ of $V_{G}$. For a real number $p>1$, define the $p$-Dirichlet sum $I_{p}(u, S)$ of $u$ by

$$
I_{p}(u, S)=\sum_{x \in S}|D u|^{p}(x),
$$

where $|D u|^{p}(x)=\sum_{y \sim x}|u(y)-u(x)|^{p}$. In particular, if $I_{p}\left(u, V_{G}\right)<\infty$, then $u$ is said to be energy finite. Define the $p$-Laplacian of $u$ at $x \in S$ by

$$
\Delta_{p} u(x)=\sum_{y \sim x}|u(y)-u(x)|^{p-2}(u(y)-u(x)) .
$$


In the case $1<p<2$, we make a convention that $|u(y)-u(x)|^{p-2}(u(y)-u(x))=$ 0 if $u(y)=u(x)$. In particular, if $\Delta_{p} u(x)=0$ for all $x \in S$, then the function $u$ is called $p$-harmonic on $S$.

Let us put

$$
T(u, \eta ; x, y)=|u(y)-u(x)|^{p-2}(u(y)-u(x))(\eta(y)-\eta(x)),
$$

whenever $u$ and $\eta$ are functions defined at $x$ and $y$. Since $|a|^{p-2} a(a-b) \geq$ $|b|^{p-2} b(a-b)$ for all real number $a \neq b$, we have

$$
T(u, u-v ; x, y) \geq T(v, u-v ; x, y)
$$

if $u$ and $v$ are defined at $x$ and $y$. The equality occurs only if $u(x)-v(x)=$ $u(y)-v(y)$.

If $S$ is a finite subset of $V_{G}$ and $h$ is a real valued function on $S \cup \partial S$, then the followings are equivalent [1]:

(i) A function $h$ is $p$-harmonic on $S$.

(ii) For any function $\eta$ on $S \cup \partial S$ such that $\eta=0$ on $\partial S$, we have

$$
\sum_{x \in S} \sum_{y \sim x} T(u, \eta ; x, y)=0 .
$$

(iii) For every function $f$ on $S \cup \partial S$ such that $f=h$ on $\partial S$, we have

$$
\sum_{x \in S}|D h|^{p}(x) \leq \sum_{x \in S}|D f|^{p}(x) .
$$

In particular, a real valued function $h$ is $p$-harmonic on $V_{G}$ if

$$
\sum_{x \in V_{G}} \sum_{y \sim x} T(u, \eta ; x, y)=0
$$

for all finitely supported real valued function $\eta$ on $V_{G}$.

In the case of $p$-harmonic functions, the comparison principle on finite subsets and the local Harnack inequality hold as follow (See [1] for the proof):

Proposition 2.1. Let $S$ be a finite subset of $V_{G}$. Suppose that $u$ and $v$ are p-harmonic on $S$ such that $u \geq v$ on $\partial S$. Then $u \geq v$ on $S$.

Proposition 2.2. Let $u$ be nonnegative on $S \cup \partial S$ and $p$-harmonic on $S$. Then for each $x \in S$, there exists a constant $C$ such that

$$
\max _{y \sim x} u(y) \leq C u(x) .
$$

For each energy finite real valued function $u$ defined on $V_{G}$, we define its norm by

$$
\|u\|_{p}=|u(o)|+I_{p}\left(u, V_{G}\right)^{1 / p}
$$

where $o$ is a fixed vertex of $V_{G}$. Let us denote $\mathcal{B D}_{p}(G)$ to be the set of all bounded energy finite functions with the norm (2). We denote by $\mathcal{B D}_{p, 0}(G)$ the closure of the set of all finitely supported functions in $\mathcal{B D}_{p}(G)$. The subset 
of all $p$-harmonic functions in $\mathcal{B D}_{p}(G)$ is denoted by $\mathcal{H B D}_{p}(G)$. Yamasaki [3] proved the discrete analogue of the Royden decomposition theorem as follows:

Proposition 2.3. For every $f \in \mathcal{B D}_{p}(G)$, there exist a unique $h \in \mathcal{H B D}_{p}(G)$ and a unique $g \in \mathcal{B D}_{p, 0}(G)$ such that $f=h+g$.

\section{Maximum principle and comparison principle}

Let us begin with defining $p$-harmonic boundary of a graph $G$ as follows: For an infinite connected graph $G$, there exists a unique (up to homeomorphism) compact Hausdorff space $\hat{G}$, called the Royden $p$-compactification of $G$, which contains $G$ as an open dense subset, every function in $\mathcal{B D}_{p}(G)$ can be continuously extended to $\hat{G}$ and the class of such extended functions separates points in $\hat{G}$. The Royden $p$-boundary of $\hat{G}$ is the set $\hat{G} \backslash G$ and denoted by $\partial \hat{G}$. The $p$-harmonic boundary $\Delta_{p, G}$ of $G$ is the subset of the Royden $p$-boundary $\partial \hat{G}$, defined by

$$
\Delta_{p, G}=\left\{\mathbf{x} \in \partial \hat{G}: f(\mathbf{x})=0 \text { for all } f \in \mathcal{B D}_{p, 0}(G)\right\} .
$$

In particular, for each subset $S$ of $V_{G}, \hat{S}$ denotes the closure of $S$ in $\hat{G}$ and $S^{d}$ denotes the double of $S$, which is obtained by identifying the interior boundaries of two copies of $S$, respectively.

Whether the closure of a subset of $\hat{G}$ intersects or not the $p$-harmonic boundary of $G$ implies how large the set of bounded energy finite functions on the subset is as follows:

Lemma 3.1. Let $\Omega$ be a subset of $V_{G}$ such that $\hat{\Omega} \cap \Delta_{p, G}=\emptyset$. Then $1 \in$ $\mathcal{B D}_{p, 0}\left(\Omega^{d}\right)$

Proof. We claim that there exists a function $w \in \mathcal{B D}_{p, 0}(G)$ such that $w>$ 1 on $\hat{\Omega}$. First of all, for each $z \in \hat{\Omega} \cap \partial \hat{G}$, since $z \notin \Delta_{p, G}$, there exists a function $f_{z} \in \mathcal{B D}_{p, 0}(G)$ such that $f_{z}(z) \neq 0$. Let us define a function $w_{z}$ on $\hat{G}$ by $w_{z}(x)=2\left|f_{z}(x)\right| /\left|f_{z}(z)\right|$. Then clearly, $w_{z}$ is a nonnegative function in $\mathcal{B D}_{p, 0}(G)$ satisfying $w_{z}(z)=2$. From the compactness of $\hat{\Omega} \cap \partial \hat{G}$, there exist finitely many points $z_{1}, z_{2}, \ldots, z_{k}$ in $\hat{\Omega} \cap \partial \hat{G}$ such that

$$
\hat{\Omega} \cap \partial \hat{G} \subset \bigcup_{j=1}^{k}\left\{x \in \hat{G}: w_{z_{j}}(x)>1\right\} .
$$

Next, for each $z \in \hat{\Omega} \backslash \cup_{j=1}^{k}\left\{x \in \hat{G}: w_{z_{j}}(x)>1\right\}$, define a function $w_{z} \in \mathcal{B D}_{p}(G)$ such that $w_{z}$ is $p$-harmonic on $N_{3}(z) \backslash N_{1}(z), w_{z}=2$ on $N_{1}(z)$, and $w_{z}=0$ on $G \backslash N_{3}(z)$. Then $w_{z} \in \mathcal{B D}_{p, 0}(G)$, since it is finitely supported. From the compactness of $\hat{\Omega} \backslash \cup_{j=1}^{k}\left\{x \in \hat{G}: w_{z_{j}}(x)>1\right\}$, there exist finitely many points $z_{k+1}, z_{k+2}, \ldots, z_{k+m}$ in $\hat{\Omega} \backslash \cup_{j=1}^{k}\left\{x \in \hat{G}: w_{z_{j}}(x)>1\right\}$ such that

$$
\left(\hat{\Omega} \backslash \bigcup_{j=1}^{k}\left\{x \in \hat{G}: w_{z_{j}}(x)>1\right\}\right) \subset \bigcup_{j=k+1}^{k+m}\left\{x \in \hat{G}: w_{z_{j}}(x)>1\right\} .
$$


Therefore, we have $w=\sum_{j=1}^{k+m} w_{z_{j}} \in \mathcal{B D}_{p, 0}(G)$ such that $w>1$ on $\hat{\Omega}$, hence the claim.

Now choose a sequence $\left\{g_{n}\right\}$ of finitely supported functions converging to $w$ in $\mathcal{B D}_{p}(G)$. Symmetrically extend $w$ and $g_{n}$ to $\Omega^{d}$, denoted again by $w$ and $g_{n}$, respectively. Clearly, each $g_{n}$ is finitely supported on $\Omega^{d}$, and $\left\{g_{n}\right\}$ converges to $w$ in $\mathcal{B D}_{p}\left(\Omega^{d}\right)$. Therefore, $w \in \mathcal{B D}_{p, 0}\left(\Omega^{d}\right)$ and $w>1$ on $\Omega^{d}$. Since $\mathcal{B D}_{p, 0}\left(\Omega^{d}\right)$ is an ideal of $\mathcal{B D}_{p}\left(\Omega^{d}\right)$, we have $1=(1 / w) w \in \mathcal{B D}_{p, 0}\left(\Omega^{d}\right)$.

We say that a connected infinite subset $\Omega$ of $V_{G}$ is $\mathcal{D}_{p}$-massive if there exists a nonnegative function $u$ on $V_{G}$ such that $u$ is $p$-harmonic on $\Omega, u=0$ on $V_{G} \backslash \Omega, \sup _{V_{G}} u=1$, and $I_{p}(u, \Omega)<\infty$. Such a function $u$ is called an inner potential of the $\mathcal{D}_{p}$-massive set $\Omega$.

We are ready to prove the maximum principle:

Theorem 3.2 (Maximum principle). Let $G$ be a graph of bounded degree and $h \in \mathcal{H B D}_{p}(G)$ such that $a \leq h \leq b$ on $\Delta_{p, G}$ for some constants $a$ and $b$. Then we have $a \leq h \leq b$ on $V_{G}$.

Proof. We first claim that $h \leq b$ on $V_{G}$. Otherwise, there exist constants $c$ and $c_{1}$ such that $b<c_{1}<c<M$, where $M=\sup _{V_{G}} h$. Let $\Omega_{c}$ be a component of the set $\left\{x \in V_{G}: h(x)>c\right\}$ with $\sup _{\Omega_{c}} h=M$. Then $V_{G} \backslash \Omega_{c}$ is nonempty from the continuity of $h$ and the fact that $h \leq b$ on $\Delta_{p, G}$.

Now construct a sequence of nonnegative functions $u_{r}$ on $V_{G}$ such that

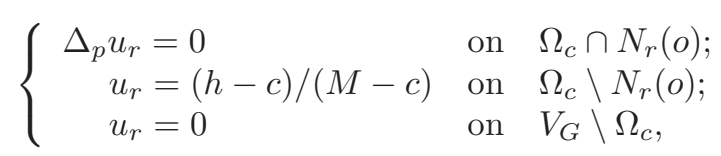

where $M=\sup _{V_{G}} h$. Then since $u_{r} \geq(h-c) /(M-c)$ on $\left(\partial \Omega_{c} \cap N_{r+1}(o)\right) \cup$ $\left(\Omega_{c} \cap \partial N_{r}(o)\right)$, by Proposition 2.1,

$$
u_{r} \geq(h-c) /(M-c) \quad \text { on } \quad \Omega_{c} \cap N_{r}(o) .
$$

On the other hand,

$$
\begin{aligned}
\left(\sum_{x \in \Omega_{c} \cap N_{r}(o)}\left|D u_{r}\right|^{p}(x)\right)^{1 / p} & \leq\left(\sum_{x \in \Omega_{c} \cap N_{r}(o)}|D((h-c) /(M-c))|^{p}(x)\right)^{1 / p} \\
& \leq \frac{1}{M-c}\left(\sum_{x \in V_{G}}|D h|^{p}(x)\right)^{1 / p} .
\end{aligned}
$$

Thus there exists a subsequence $\left\{u_{r_{k}}\right\}$ converging to a nonnegative function $u$ on $V_{G}$ such that $u$ is $p$-harmonic on $\Omega_{c}, u=0$ on $V_{G} \backslash \Omega_{c}$, $\sup _{V_{G}} u=1$, and $I_{p}\left(u, \Omega_{c}\right)<\infty$. This implies that $\Omega_{c}$ is $\mathcal{D}_{p}$-massive with an inner potential $u$.

Let $O_{\epsilon}=\left\{x \in \Omega_{c}: u(x)>1-\epsilon\right\}$ for $0<\epsilon<1$. Then by Proposition 2.2, there exists a sufficiently small $\epsilon>0$ such that

$$
d\left(\partial \Omega_{c}, O_{\epsilon}\right) \geq 2 .
$$


Let $D_{\epsilon}$ be a component of the set $\left\{x \in \Omega_{c}:(h(x)-c) /(M-c)>1-\epsilon\right\}$ such that $\sup _{D_{\epsilon}} h=M$. Then $O_{\epsilon} \supset D_{\epsilon}$. On the other hand, since $u \geq(h-c) /(M-c)$ on $\Omega_{c}$, we have

$$
d\left(\partial \Omega_{c}, D_{\epsilon}\right) \geq 2 .
$$

Let $\Omega=\left\{x \in V_{G}: h(x)>c_{1}\right\}$, then $\hat{\Omega} \cap \Delta_{p, G}=\emptyset$. Thus by Lemma 3.1, there exists a sequence $\left\{\phi_{n}\right\}$ of finitely supported nonnegative functions converging to 1 in $\mathcal{B D}_{p}\left(\Omega^{d}\right)$. Let $v=\max \{h-c, 0\}$, then $v=h-c$ on $\Omega_{c}$. In particular, since $d\left(\partial \Omega_{c}, D_{\epsilon}\right) \geq 2, D_{\epsilon} \cup \partial D_{\epsilon} \subset \Omega_{c}$. Hence, $v=h-c$ on $D_{\epsilon} \cup \partial D_{\epsilon}$. On the other hand, the function $v \phi_{n}$ vanishes on $V_{G} \backslash\left(\Omega \cap K_{n}\right)$, where $K_{n}$ is the support of $\phi_{n}$ in $\Omega^{d}$, and $\left\{v \phi_{n}\right\}$ converges to $v$ with the norm

$$
\|f\|_{p}=\left|f\left(x_{0}\right)\right|+\left(\sum_{x \in D_{\epsilon}}|D f|^{p}(x)\right)^{1 / p}
$$

where $x_{0}$ is a fixed point in $D_{\epsilon}$. Thus we get

$$
\begin{aligned}
& \sum_{x \in D_{\epsilon}} \sum_{y \sim x}|h(y)-h(x)|^{p} \\
= & \sum_{x \in D_{\epsilon}} \sum_{y \sim x}|h(y)-h(x)|^{p-2}(h(y)-h(x))(h(y)-h(x)) \\
= & \sum_{x \in D_{\epsilon}} \sum_{y \sim x}|h(y)-h(x)|^{p-2}(h(y)-h(x))(v(y)-v(x)) \\
= & \lim _{n \rightarrow \infty}\left(\sum_{x \in D_{\epsilon}} \sum_{y \sim x}|h(y)-h(x)|^{p-2}(h(y)-h(x))\left(\left(v \phi_{n}\right)(y)-\left(v \phi_{n}\right)(x)\right)\right) \\
= & 0 .
\end{aligned}
$$

Therefore, $h$ is constant on $D_{\epsilon}$ such that $h \equiv M$ on $D_{\epsilon}$. From the $p$-harmonicity of $h$ and the connectedness of $\Omega_{c}$, we have $h \equiv M$ on $\Omega_{c}$. Also, from the $p$ harmonicity of $h$ and the connectedness, we conclude that $h \equiv M$ on $\partial \Omega_{c}$. However, this is impossible since $h \leq c$ on $\partial \Omega_{c}$. Consequently, we have the claim that $h \leq b$ on $V_{G}$.

Similarly arguing, we get the remains.

By using the maximum principle, we can give a duality relation between $\mathcal{B D}_{p, 0}(G)$ and $\Delta_{p, G}$ as follows:

Proposition 3.3. $\mathcal{B D}_{p, 0}(G)=\left\{f \in \mathcal{B D}_{p}(G): f=0\right.$ on $\left.\Delta_{p, G}\right\}$.

Proof. Suppose that $f \in \mathcal{B D}_{p}(G)$ with $\left.f\right|_{\Delta_{p, G}}=0$. By Proposition 2.3, there exist $h \in \mathcal{H B D}_{p}(G)$ and $g \in \mathcal{B D}_{p, 0}(G)$ such that $f=h+g$. Since $\left.g\right|_{\Delta_{p, G}}=0$, $\left.h\right|_{\Delta_{p, G}}=0$. By the maximum principle, we have $h \equiv 0$ on $V_{G}$, hence $f=g \in$ $\mathcal{B D}_{p, 0}(G)$.

By modifying the program of Holopainen and Soardi [1] and applying the duality relation Proposition 3.3, we prove the comparison principle for $p$-harmonic functions in terms of $p$-harmonic boundary as follows: 
Theorem 3.4 (Comparison principle). Let $G$ be a graph of bounded degree and $u, v \in \mathcal{H B D}_{p}(G)$ such that $v \leq u$ on $\Delta_{p, G}$. Then we have $v \leq u$ on $V_{G}$.

Proof. Let $U^{+}=\left\{x \in V_{G}: v(x) \leq u(x)\right\}$ and $U^{-}=\left\{x \in V_{G}: v(x)>u(x)\right\}$. For each vertex $x \in V_{G}$, we set $U_{x}^{+}=\{y \sim x: v(y) \leq u(y)\}$ and $U_{x}^{-}=\{y \sim$ $x: v(y)>u(y)\}$.

Let $\eta=\max \{v-u, 0\}$. Then $\eta \geq 0$ on $V_{G}, \eta=0$ on $\Delta_{p, G}$, and $\eta(y)=0$ for all $y \in U^{+}$and $y \in U_{x}^{+}$. By Proposition 3.3, we have $\eta \in \mathcal{B D}_{p, 0}(G)$, hence we get

$$
\begin{aligned}
0 & =\sum_{x \in V_{G}} \sum_{y \sim x}(T(v, \eta ; x, y)-T(u, \eta ; x, y)) \\
& =I+I I+I I I
\end{aligned}
$$

where

$$
\begin{aligned}
I= & \sum_{x \in U^{+}} \sum_{y \in U_{x}^{+}}(T(v, \eta ; x, y)-T(u, \eta ; x, y)), \\
I I= & \sum_{x \in U^{+}} \sum_{y \in U_{x}^{-}}(T(v, \eta ; x, y)-T(u, \eta ; x, y)) \\
& +\sum_{x \in U^{-}} \sum_{y \in U_{x}^{+}}(T(v, \eta ; x, y)-T(u, \eta ; x, y)), \\
I I I= & \sum_{x \in U^{-}} \sum_{y \in U_{x}^{-}}(T(v, \eta ; x, y)-T(u, \eta ; x, y)) .
\end{aligned}
$$

First of all, we have $I=0$ since $\eta(y)=0$ for all $y \in U^{+}$and $y \in U_{x}^{+}$. Secondly, by (1) we get $I I I \geq 0$ since $\eta=v-u$ on $U^{-}$or on $U_{x}^{-}$.

We claim that if there exists at least one pair $x, y$ such that $x \in U^{+}$and $y \in U_{x}^{-}$, or $x \in U^{-}$and $y \in U_{x}^{+}$, then $I I>0$. However, since $0=I+I I+I I I$, it is impossible. Thus for any pair $x, y$ with $y \sim x$,

$$
\text { either } v(x) \leq u(x), v(y) \leq u(y) \quad \text { or } \quad v(x)>u(x), v(y)>u(y) .
$$

If for any pair $x, y$ with $y \sim x, v(x)>u(x), v(y)>u(y)$, then since $G$ is connected, $v>u$ on $V_{G}$. Let $w=v-u$. Then $w>0$ on $V_{G}$, hence $w \geq 0$ on $\Delta_{p, G}$. On the other hand, since $w \leq 0$ on $\Delta_{p, G}$ from the hypothesis, we have $w=0$ on $\Delta_{p, G}$. By Proposition 3.3, we have $w \in \mathcal{B D}_{p, 0}(G)$, hence we get

$$
0=\sum_{x \in V_{G}} \sum_{y \sim x}(T(v, w ; x, y)-T(u, w ; x, y)) \text {. }
$$

By (1), this implies that $v-u \equiv c$ on $V_{G}$ for some constant $c$. Since $v \leq u$ on $\Delta_{p, G}$, we conclude that $c \leq 0$. However, this is a contradiction to the fact that $v>u$ on $V_{G}$. Therefore, for any pair $x, y$ with $y \sim x$, we have $v(x) \leq u(x), v(y) \leq u(y)$, hence we have the consequence $v \leq u$ on $V_{G}$.

We now prove the claim. Suppose that there exists a pair $x, y$ such that $x \in U^{+}$and $y \in U_{x}^{-}$. Then by the definition of $\eta$, we have $\eta(y)-\eta(x)=$ 
$v(y)-u(y)>0$. Since

$$
\begin{aligned}
& T(v, \eta ; x, y)-T(u, \eta ; x, y) \\
= & (v(y)-u(y))\left(|v(y)-v(x)|^{p-2}(v(y)-v(x))-|u(y)-u(x)|^{p-2}(u(y)-u(x))\right),
\end{aligned}
$$

we have only to prove that

$$
|v(y)-v(x)|^{p-2}(v(y)-v(x))-|u(y)-u(x)|^{p-2}(u(y)-u(x))>0 .
$$

Since $v(y)-u(y)>0 \geq v(x)-u(x)$, we have $v(y)-v(x)>u(y)-u(x)$. If $u(y)-u(x) \geq 0$, then

$$
\begin{aligned}
& |v(y)-v(x)|^{p-2}(v(y)-v(x))-|u(y)-u(x)|^{p-2}(u(y)-u(x)) \\
= & (v(y)-v(x))^{p-1}-(u(y)-u(x))^{p-1}>0 .
\end{aligned}
$$

In the case that $u(y)-u(x)<0$ and $v(y)-v(x)>0$, we have

$$
\begin{aligned}
& |v(y)-v(x)|^{p-2}(v(y)-v(x))-|u(y)-u(x)|^{p-2}(u(y)-u(x)) \\
= & |v(y)-v(x)|^{p-1}+|u(y)-u(x)|^{p-1}>0 .
\end{aligned}
$$

In the other case $u(y)-u(x)<0$ and $v(y)-v(x) \leq 0$, we have $|v(y)-v(x)|<$ $|u(y)-u(x)|$. Hence

$$
\begin{aligned}
& |v(y)-v(x)|^{p-2}(v(y)-v(x))-|u(y)-u(x)|^{p-2}(u(y)-u(x)) \\
= & -|v(y)-v(x)|^{p-1}+|u(y)-u(x)|^{p-1}>0 .
\end{aligned}
$$

Similarly arguing as above, for any pair $x, y$ such that $x \in U^{-}$and $y \in U_{x}^{+}$, we have

$$
\begin{aligned}
& T(v, \eta ; x, y)-T(u, \eta ; x, y) \\
= & -(v(x)-u(x))\left(|v(y)-v(x)|^{p-2}(v(y)-v(x))-|u(y)-u(x)|^{p-2}(u(y)-u(x))\right) \\
> & 0 .
\end{aligned}
$$

Therefore, we have the claim.

As an application of the comparison principle, we prove the solvability of the boundary value problem of $p$-harmonic functions in terms of $p$-harmonic boundary of graphs as follows:

Theorem 3.5. Let $G$ be a graph of bounded degree. Then for any continuous function $f$ on $\Delta_{p, G}$, there exists a unique p-harmonic function $h$, which is a limit of a sequence of functions in $\mathcal{H B D}_{p}(G)$ in the sense of supremum norm, such that

$$
h(\mathbf{x})=f(\mathbf{x})
$$

for all $\mathrm{x} \in \Delta_{p, G}$. 
Proof. Let $f: \Delta_{p, G} \rightarrow \mathbb{R}$ be a continuous function. Then there is a sequence $\left\{f_{n}\right\}$ in $\mathcal{B D}_{p}(G)$ such that

$$
\lim _{n \rightarrow \infty} \sup _{V_{G}}\left|f_{n}-\hat{f}\right|=0,
$$

where $\hat{f}=\lim _{n \rightarrow \infty} f_{n}$ and $\left.\hat{f}\right|_{\Delta_{p, G}}=f$. For each $n \in \mathbb{N}$, define a continuous function $h_{n, r}$ on $V_{G}$ such that

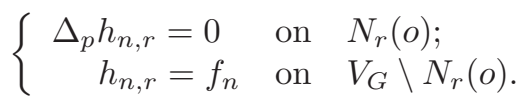

Obviously, $h_{n, r} \in \mathcal{B D}_{p}(G)$ and $\left|h_{n, r}\right| \leq \sup _{V_{G}}\left|f_{n}\right|$ on $V_{G}$. Thus there exists a subsequence $\left\{h_{n, r_{k}}\right\}$ converging uniformly to a limit function $h_{n}$ on any finite subset of $V_{G}$. In particular, $h_{n}$ is a $p$-harmonic function and $\left\{h_{n, r_{k}}\right\}$ converges to $h_{n}$ in $\mathcal{B D}_{p}(G)$. Hence $h_{n} \in \mathcal{H B D}_{p}(G)$, and by Proposition 2.3, there exists a unique $g_{n} \in \mathcal{B D}_{p, 0}(G)$ such that $f_{n}=h_{n}+g_{n}$.

On the other hand, from (4), for any given $\epsilon>0$, there is $N \in \mathbb{N}$ such that if $n, m \geq N$,

$$
\sup _{V_{G}}\left|f_{n}-f_{m}\right|<\epsilon,
$$

hence

$$
\sup _{\partial N_{r}(o)}\left|h_{n, r}-h_{m, r}\right|<\epsilon
$$

for all $r \in \mathbf{N}$. Then by Proposition 2.1, we get

$$
\sup _{N_{r}(o)}\left|h_{n, r}-h_{m, r}\right|<\epsilon
$$

for all $r>0$. This yields

$$
\sup _{S}\left|h_{n}-h_{m}\right|<3 \epsilon
$$

for any finite subset $S$ of $V_{G}$, hence

$$
\sup _{M}\left|h_{n}-h_{m}\right| \leq 3 \epsilon .
$$

Therefore, $\left\{h_{n}\right\}$ converges uniformly to a limit function $h$ on $V_{G}$ and $h$ is $p$ harmonic on $V_{G}$.

Now for given $\epsilon>0$, choose $N \in \mathbb{N}$ such that for all $n \geq N$,

$$
\sup _{V_{G}}\left|f_{n}-\hat{f}\right|<\epsilon \quad \text { and } \quad \sup _{V_{G}}\left|h_{n}-h\right|<\epsilon \text {. }
$$

Since $h_{n} \equiv f_{n}$ on $\Delta_{p, G}$ and $\epsilon>0$ is arbitrarily chosen, we have

$$
h(\mathbf{x})=f(\mathbf{x})
$$

for all $\mathrm{x} \in \Delta_{p, G}$.

Suppose that $h$ and $\tilde{h}$ are $p$-harmonic functions satisfying (3), and $\left\{h_{n}\right\}$ and $\left\{\tilde{h}_{n}\right\}$ are sequences in $\mathcal{H B D}_{p}(G)$ such that

$$
\lim _{n \rightarrow \infty} \sup _{V_{G}}\left|h_{n}-h\right|=0 \quad \text { and } \lim _{n \rightarrow \infty} \sup _{V_{G}}\left|\tilde{h}_{n}-\tilde{h}\right|=0 .
$$


Then for any $\epsilon>0$, there is an $N \in \mathbb{N}$ such that for any $n \geq N$,

$$
\sup _{V_{G}}\left|h_{n}-h\right|<\epsilon \quad \text { and } \sup _{V_{G}}\left|\tilde{h}_{n}-\tilde{h}\right|<\epsilon .
$$

Since $h(\mathbf{x})=f(\mathbf{x})=\tilde{h}(\mathbf{x})$ for all $\mathbf{x} \in \Delta_{p, G}$, we have for any $n \geq N$,

$$
\tilde{h}_{n}(\mathbf{x})-2 \epsilon<h_{n}(\mathbf{x})<\tilde{h}_{n}(\mathbf{x})+2 \epsilon
$$

for all $\mathrm{x} \in \Delta_{p, G}$. By Theorem 3.2,

$$
\tilde{h}_{n}-2 \epsilon<h_{n}<\tilde{h}_{n}+2 \epsilon \text { on } V_{G} .
$$

From (5), we have

$$
\sup _{V_{G}}|h-\tilde{h}|<4 \epsilon .
$$

Since $\epsilon>0$ is arbitrarily chosen, we have $h=\tilde{h}$ on $V_{G}$.

\section{References}

[1] I. Holopainen and P. M. Soardi, p-harmonic functions on graphs and manifolds, Manuscripta Math. 94 (1997), no. 1, 95-110.

[2] J.-H. Kim and S.-Y. Chung, Comparison principles for the p-Laplacian on nonlinear networks, J. Difference Equ. Appl. 16 (2010), no. 10, 1151-1163.

[3] M. Yamasaki, Ideal boundary limit of discrete Dirichlet functions, Hiroshima Math. J. 16 (1986), no. 2, 353-360.

Department of Mathematics Education

EWHA WOMANS UNIVERSITY

SEOUl 120-750, KorEA

E-mail address: yonghah@ewha.ac.kr 